Open Access

\title{
Ultrasonographic diagnosis of congenital membranous jejunal stenosis and gastric duplication cyst in a newborn: a case report

\author{
Liu Qinghua ${ }^{1 *}$,Wu Shoucai ${ }^{2}$, Liu Xiaofang ${ }^{1}$, Zhang Xincun ${ }^{1}$ and Miao Lili ${ }^{1}$
}

\begin{abstract}
Introduction: Congenital jejunal stenosis and gastric duplication cysts are very rare congenital anomalies of the gastrointestinal tract in the newborn. We present a case of congenital membranous jejunal stenosis associated with gastric duplication cysts, which was diagnosed by ultrasonography. To the best of our knowledge, this is the first report of ultrasonographic diagnosis of congenital membranous jejunal stenosis associated with a gastric duplication cyst in a newborn.
\end{abstract}

Case presentation: A 1-month-old Chinese baby girl presented with projectile vomiting and hyperpyrexia for 3 days. An upper gastrointestinal contrast study showed incomplete duodenal obstruction; however, ultrasonography revealed congenital membranous jejunal stenosis associated with a gastric duplication cyst. After surgical excision of the jejunal membrane and gastric duplication cyst, she recovered well with no bilious vomiting at the 1-year follow-up.

Conclusion: Ultrasonography is a useful tool for the evaluation of membranous jejunal stenosis and the identification of small, asymptomatic gastrointestinal duplication cysts.

Keywords: Congenital membranous jejunal stenosis, Gastric duplication cyst, Newborn, Ultrasonography

\section{Introduction}

Congenital jejunal stenosis and gastric duplication cysts are very rare congenital anomalies of the gastrointestinal (GI) tract in the newborn; the prevalence rate of jejunoileal atresia and stenosis has been reported to be between 1 in 330 and 0.9 in 10,000 live births, and stenosis occurs in $7 \%$ of cases [1]. The incidence of duplication cysts of the alimentary system is approximately 1 in 4500 live births [2], and gastric duplication cysts represent only 4 to $5 \%$ of these cases $[3,4]$. We present a case of congenital membranous jejunal stenosis associated with gastric duplication cysts, which was diagnosed by ultrasonography. The patient underwent surgery and recovered well. To the best of our knowledge, this is the first report of ultrasonographic diagnosis of congenital membranous jejunal stenosis associated with a gastric duplication cyst in a newborn.

\footnotetext{
* Correspondence: xr6547@163.com

'Department of Ultrasound, Jinan Children's Hospital, Jinan 250022, China Full list of author information is available at the end of the article
}

\section{Case presentation}

A 1-month-old Chinese baby girl presented to our emergency department with vomiting and high-grade fever, which had occurred for 3 days. The fever was not associated with rigors and chills. She presented with a history of intermittent projectile and bilious vomiting after breastfeeding. Vomiting occurred approximately five times each day, at a volume of $10 \mathrm{~mL}$ at each instance. A general physical examination revealed a temperature of $39^{\circ} \mathrm{C}$, pulse rate of 100 /minute, and respiratory rate of 30/minute. On abdominal examination, no abdominal distention, regional tenderness, or rebound was observed, and no masses or viscera could be palpated. Bowel sounds were audible, and other physical examination results were normal. An upper GI contrast study in another hospital showed incomplete duodenal obstruction (Fig. 1). However, to clarify the cause of intestinal obstruction, she subsequently underwent ultrasonographic examination after hospitalization. Ultrasonography showed continuous opening of the pylorus and significant dilatation of her duodenum; her upper jejunum in the left abdomen was approximately $4 \mathrm{~cm}$ in diameter, and we observed frequent reverse peristalsis. 


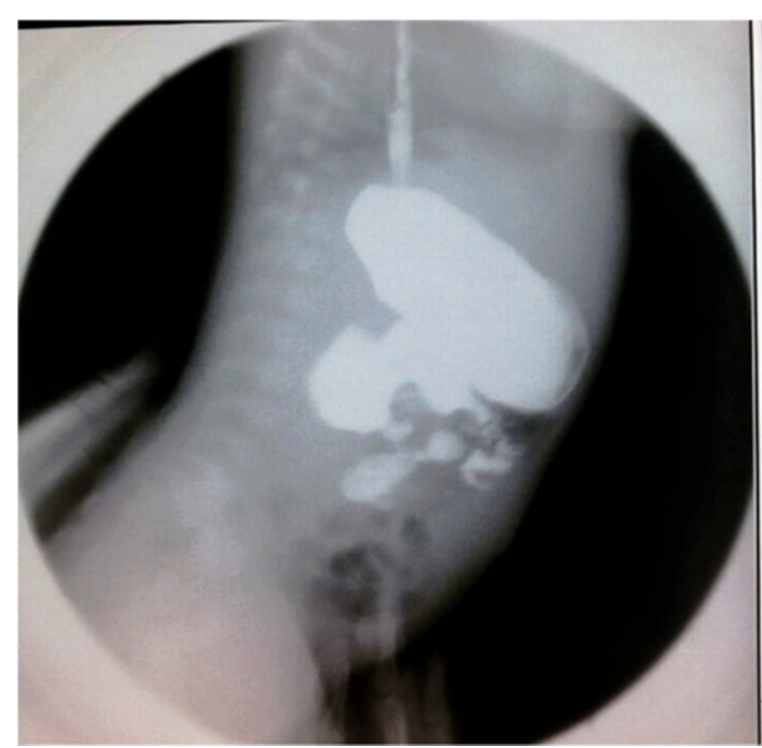

Fig. 1 Upper gastrointestinal contrast study showing marked dilatation of the duodenum

An intraluminal membrane was visible in the dilated distal jejunum site (Fig. 2), and the "billow" in the proximal jejunum could be seen to intermittently move in and out of a pinhole in the mucosal diaphragm. The distal intestine was deflated, and there was an absence of gas; by ultrasonography, a diagnosis of congenital membranous jejunal stenosis was considered. Furthermore, an ultrasound showed a cystic structure sized $2.0 \times 1.5 \mathrm{~cm}$ in the greater curvature in the left hypochondrium (Fig. 3). It was firmly attached at the gastric wall, and the cyst walls consisted of an inner echogenic rim and an outer echogenic rim area. The central area was hypoechoic. A gastric duplication cyst was considered the most likely diagnosis, and a laparotomy was planned after initial stabilization. During surgery, jejunal stenosis situated approximately $15 \mathrm{~cm}$ from the ligament of Treitz and a diaphragm with a pinhole in the intestinal cavity was found and resected. In addition, surgical excision of the cyst located on the greater curvature of the stomach was performed. A histopathological examination revealed that both sides of the resected intestinal membrane were covered with intestinal mucosa (Fig. 4) and the structure of the gastric mucosa and smooth muscles in the wall of the cyst, confirming the diagnosis of

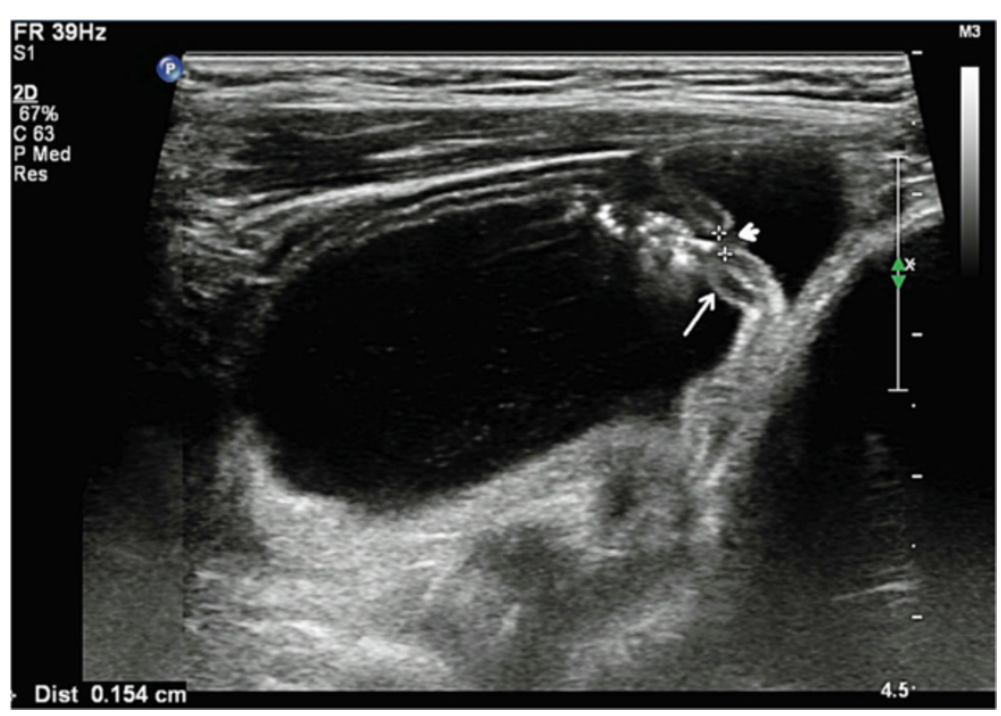

Fig. 2 Ultrasonography showing dilatation of the duodenum and proximal jejunum. A membrane (arrow) and a pinhole (small arrowhead) are seen in the jejunal membrane 


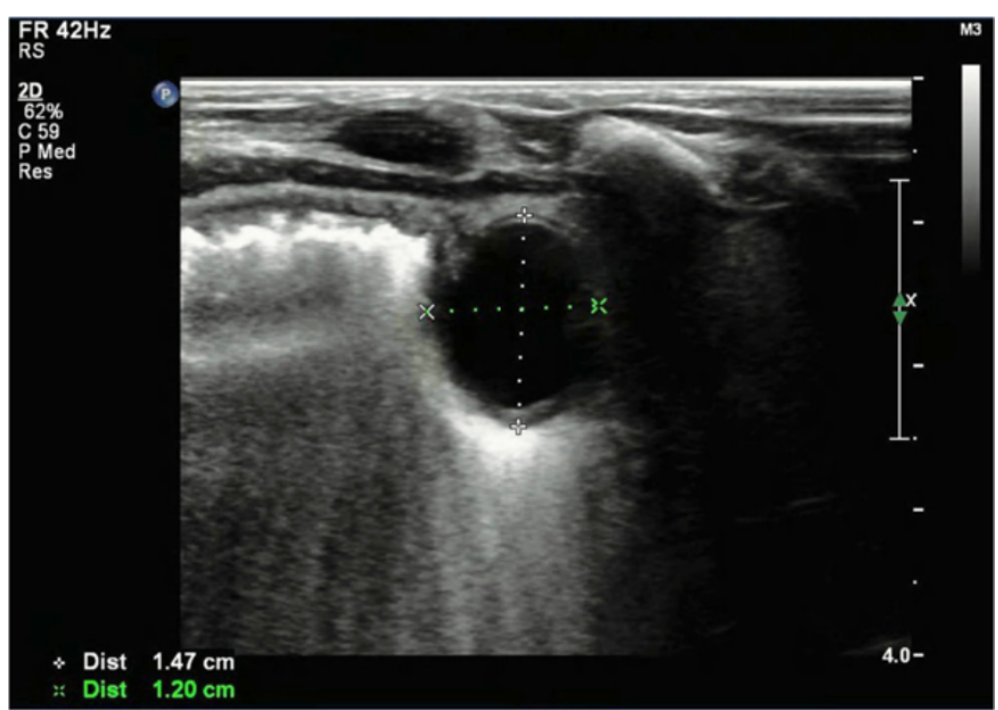

Fig. 3 Ultrasonographic image of the gastric duplication cyst. It was $1.5 \times 1.4 \mathrm{~cm}$ and intimately attached at the greater curvature of the stomach

membranous jejunal stenosis and gastric duplication cyst (Fig. 5). Postoperative recovery was uneventful, and she was discharged on the ninth day following surgery. She recovered well with no bilious vomiting at the 1-year follow-up.

\section{Discussion}

In general, the symptoms of patients with congenital anomalies of the alimentary tract present early after birth with recurrent, progressive vomiting. Bilious vomiting indicates obstruction distal to the ampulla of Vater [5], which requires an immediate and correct diagnosis and surgical correction to avoid serious complications. Normally, within 3 hours of birth, the entire small bowel contains gas, whereas gas is observed in the sigmoid colon 8 to 9 hours after birth [6]. In some patients with congenital anomalies of the GI tract causing obstructions, the proximal bowel with lesions exhibits dilatation and effusion, and the distal intestinal cavity has little or no gas, thereby creating favorable conditions for ultrasonic detection of the lesions. Furthermore, ultrasonography of the GI tract is noninvasive, simple, radiation-free, and fairly accurate for the diagnosis of GI obstruction. Therefore, ultrasonography plays a vital role in the evaluation of patients with congenital anomalies of the GI tract. Ultrasonography has been used as an alternative to barium studies in diagnosing certain congenital anomalies of the GI tract, including annular pancreas, hypertrophic pyloric stenosis, enteric duplication cysts, malrotation, and midgut volvulus [5]. However, the use of ultrasonography for the detection and diagnosis of membranous jejunal stenosis has not been previously reported in the literature. A previous study reported that jejunoileal atresia and stenosis are difficult to evaluate [7]. In the present case, ultrasonography revealed the mucosal diaphragm with a pinhole in the jejunum, the duodenum and proximal jejunum loops significantly dilated with fluid, and the distal small intestine deflated with little gas. Based on this case, ultrasonography may have greater importance in the evaluation of membranous jejunal stenosis, as it enables the distinction from atresia and provides excellent anatomic details for correct diagnosis and treatment planning.

Duplication cysts are rare congenital anomalies that can occur in any part of the GI tract, although the most common site is the ileum [3]. Gastric duplication cysts represent only 4 to $5 \%$ of duplication cysts and are located along the greater curvature of the stomach, usually in the antrum [3, 4]. Possible causes of gastric duplication cysts

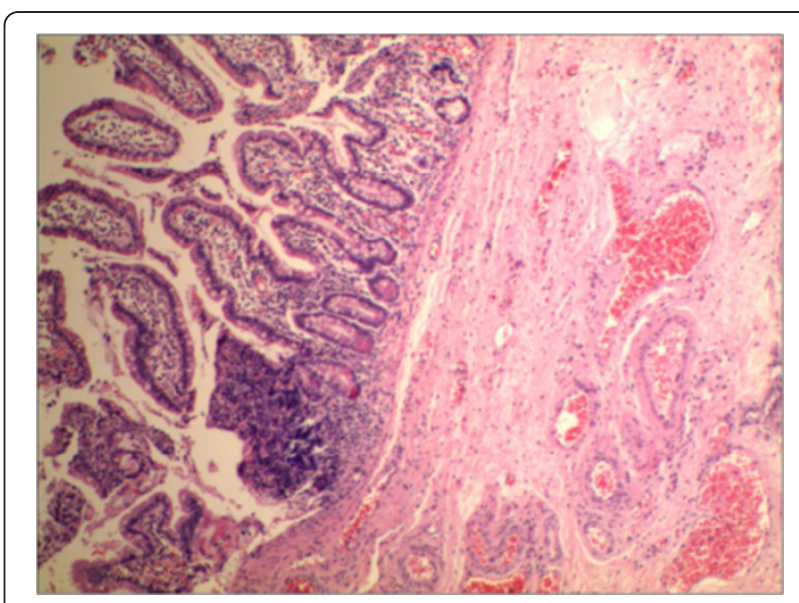

Fig. 4 Histopathological examination showing both sides of the resected intestinal membrane covered with intestinal mucosa 


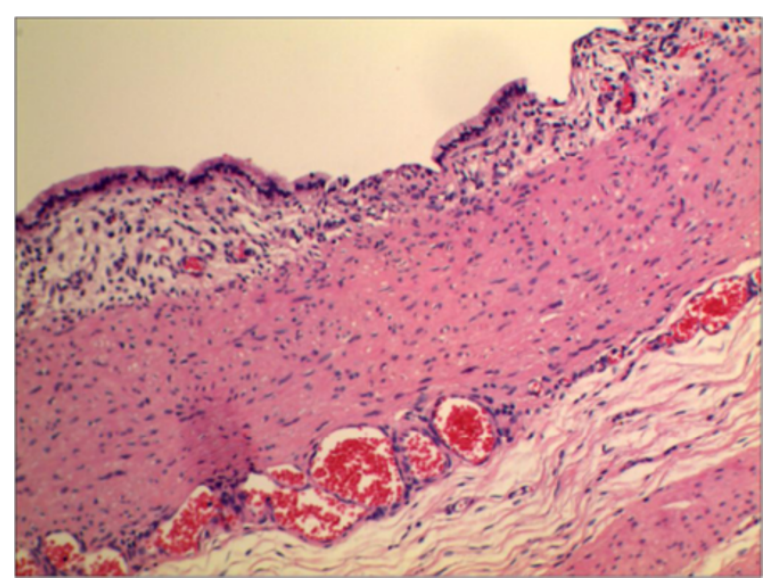

Fig. 5 Histological examination of the resected cyst. Gastric mucosa and smooth muscles are seen in the wall of the cyst

include errors in recanalization of the alimentary tract, McLetchie's theory of traction diverticulum formation caused by endo-ectodermal adhesions, split notochord, and partial twinning $[8,9]$. On clinical examination, gastric duplication cysts can present with vague abdominal pain, vomiting, and occasionally a palpable abdominal mass; however, presentation is usually asymptomatic and therefore difficult to diagnose [10]. In the present case, a gastric duplication cyst was incidentally diagnosed by abdominal ultrasonography. Ultrasonography showed a small cystic lesion on the greater curvature of the stomach in the left hypochondrium. Characteristic ultrasonographic features of a GI duplication cyst are a hypoechoic muscular rim surrounding an echogenic mucosa; these features are specific to gastric duplication cysts and are not seen with other cystic lesions [11]. However, in the present case, an upper GI tract contrast study was performed, with no evidence of mass lesions. This case report illustrates the important role of ultrasonography in detecting small, unsuspected duplication cysts.

\section{Conclusions}

Using ultrasonography, we successfully diagnosed congenital membranous jejunal stenosis associated with a gastric duplication cyst in a newborn. This case emphasizes that ultrasonography in a baby with congenital jejunal stenosis was effective as a diagnostic modality. In addition, ultrasonography can be helpful for the accurate identification of small and asymptomatic GI duplication cysts. Ultrasonography is superior to radiography for revealing the cause of some intestinal obstructions, suggesting that babies with bilious vomiting should have a routine ultrasound for diagnosis.

\section{Consent}

Written informed consent was obtained from the patient's legal guardian for publication of this case report and accompanying images. A copy of the written consent is available for review by the Editor-in-Chief of this journal.

\section{Abbreviation}

Gl: Gastrointestinal.

\section{Competing interests}

The authors declare that they have no competing interests.

\section{Authors' contributions}

LQ performed the ultrasonographic examination of the case, and was a major contributor in writing the manuscript. WS obtained the relevant literature. LX collected the patient's clinical data. ZX and ML translated and proofread the manuscript in English. All authors read and approved the manuscript.

\section{Acknowledgments}

We are very grateful to our supervisor, Editage, and Dr Zheng Wenhui, who provided valuable guidance at every stage of the writing of this report.

\section{Author details}

${ }^{1}$ Department of Ultrasound, Jinan Children's Hospital, Jinan 250022, China. ${ }^{2}$ Department of Ultrasound, Qilu Hospital, Shandong University, Jinan 250012, China.

Received: 24 February 2015 Accepted: 29 June 2015

Published online: 28 July 2015

\section{References}

1. Stollman TH, de Blaauw I, Wijnen MH, van der Staak FH, Rieu PN, Draaisma $J M$, et al. Decreased mortality but increased morbidity in neonates with jejunoileal atresia; a study of 114 cases over a 34-year period. J Pediatr Surg. 2009:44:217-21.

2. Potter EL. Pathology of the fetus and newborn. 2nd ed. Chicago: Year Book Medical Publishers; 1961.

3. Faerber EN, Balsara R, Vinocur CD, de Chadarevian JP. Gastric duplication with hemoptysis: CT findings. AJR Am J Roentgenol. 1993;161:1245-6.

4. Sieunarine K, Manmohansingh E. Gastric duplication cyst presenting as an acute abdomen in a child. J Pediatr Surg. 1989;24:1152.

5. Gupta AK, Guglani B. Imaging of congenital anomalies of the gastrointestinal tract. Indian J Pediatr. 2005;72:403-14.

6. Berrocal T, Lamas M, Gutieérrez J, Torres I, Prieto C, del Hoyo ML. Congenital anomalies of the small intestine, colon, and rectum. Radiographics. 1999;19:1219-36.

7. Sunada K, Yamamoto H, Kita H, Yano T, Sato H, Hayashi Y, et al. Clinical outcomes of enteroscopy using the double-balloon method for strictures of the small intestine. World J Gastroenterol. 2005;11:1087-9. 
8. Bonacci JL, Schlatter MG. Gastric duplication cyst: a unique presentation. J Pediatr Surg. 2008;43:1203-5.

9. Shinde T, Lindner J, Silverman J, Agrawal R, Dhawan M. Gastric-duplication cyst with an aberrant pancreatic-ductal system: an unusual cause of recurrent abdominal pain. Gastrointest Endosc. 2009;69:377-9.

10. Prinsloo H, Loveland J, Grieve A, Andronikou S, Valli OM. Gastric duplication cysts as a rare cause of haematemesis: diagnostic challenges in two children. Pediatr Surg Int. 2011;27:1127-30.

11. Barr LL, Hayden Jr CK, Stransberry SD, Swischuk LE. Enteric duplication cysts in children: are their ultrasonographic wall characteristics diagnostic? Pediatr Radiol. 1990;20:326-8.

\section{Submit your next manuscript to BioMed Central} and take full advantage of:

- Convenient online submission

- Thorough peer review

- No space constraints or color figure charges

- Immediate publication on acceptance

- Inclusion in PubMed, CAS, Scopus and Google Scholar

- Research which is freely available for redistribution 